\title{
APPROXIMATING A NORM BY A POLYNOMIAL
}

\author{
Alexander Barvinok
}

November 2, 2018

\begin{abstract}
We prove that for any norm $\|\cdot\|$ in the $d$-dimensional real vector space $V$ and for any odd $n>0$ there is a non-negative polynomial $p(x), x \in V$ of degree $2 n$ such that

$$
p^{\frac{1}{2 n}}(x) \leq\|x\| \leq\left(\begin{array}{c}
n+d-1 \\
n
\end{array}\right)^{\frac{1}{2 n}} p^{\frac{1}{2 n}}(x) .
$$

Corollaries and polynomial approximations of the Minkowski functional of a convex body are discussed.
\end{abstract}

\section{Introduction and the Main Result}

Our main motivation is the following general question. Let us fix a norm $\|\cdot\|$ in a finite dimensional real vector space $V$ (or, more generally, the Minkowski functional of a convex body in $V$ ). Given a point $x \in V$, how fast can one compute or approximate $\|x\|$ ? For example, various optimization problems can be posed this way. As is well known, (see, for example, Lecture 3 of [1]), any norm in $V$ can be approximated by an $\ell^{2}$ norm in $V$ within a factor of $\sqrt{\operatorname{dim} V}$. From the computational complexity point of view, an $\ell^{2}$ norm of $x$ is just the square root of a positive definite quadratic form $p$ in $x$ and hence can be computed "quickly", that is, in time polynomial in $\operatorname{dim} V$ for any $x \in V$ given by its coordinates in some basis of $V$. Note, that we do not count the time required for "preprocessing" the norm to obtain the quadratic form $p$, as we consider the norm fixed and not a part of the input. It turns out that by employing higher degree forms $p$, we can improve the approximation: for any $c>0$, given an $x \in V$, one can approximate $\|x\|$ within a factor of $c \sqrt{\operatorname{dim} V}$ in time polynomial in $\operatorname{dim} V$. This, and some other approximation results follow easily from our main theorem.

Key words and phrases. norm, approximation, polynomial, John's ellipsoid, computational complexity.

This research was partially supported by NSF Grant DMS 9734138.

Typeset by $\mathcal{A} \mathcal{M S}-\mathrm{T}_{\mathrm{E}} \mathrm{X}$ 
(1.1) Theorem. Let $V$ be a d-dimensional real vector space and let $\|\cdot\|: V \longrightarrow \mathbb{R}$ be a norm in $V$. For any odd integer $n>0$ there exists a homogeneous polynomial $p: V \longrightarrow \mathbb{R}$ of degree $2 n$ such that $p(x) \geq 0$ and

$$
p^{\frac{1}{2 n}}(x) \leq\|x\| \leq\left(\begin{array}{c}
n+d-1 \\
n
\end{array}\right)^{\frac{1}{2 n}} p^{\frac{1}{2 n}}(x)
$$

for all $x \in V$.

We prove Theorem 1.1 in Section 2.

Let us fix an $n$ in Theorem 1.1. Then, as $d$ grows, the value of $p^{\frac{1}{2 n}}(x)$ approximates $\|\cdot\|$ within a factor of $c_{n} \sqrt{d}$, where $c_{n} \approx(n !)^{-\frac{1}{2 n}} \approx \sqrt{e / n}$. Since for any fixed $n$, computation of $p(x)$ takes a $d^{O(n)}$ time, for any $c>0$ we obtain a polynomial time algorithm to approximate $\|x\|$ within a factor of $c \sqrt{d}$ (again, we do not count the time required for preprocessing, that is, to find the polynomial $p$ ).

If we allow $n$ to grow linearly with $d$, we can get a constant factor approximation. Indeed, if we choose $n=\gamma d$ for some $\gamma>0$, for large $d$ we have

$$
\left(\begin{array}{c}
n+d-1 \\
n
\end{array}\right)^{\frac{1}{2 n}} \approx \exp \left\{\frac{1}{2} \ln \frac{\gamma+1}{\gamma}+\frac{1}{2 \gamma} \ln (\gamma+1)\right\} .
$$

Since for any fixed $\gamma>0$, computation of $p(x)$ takes $2^{O(d)}$ time, for any $c>1$ we can get an algorithm of $2^{O(d)}$ complexity approximating the value of $\|x\|$ within a factor of $c$.

One can hope that for special norms $\|\cdot\|$ (for example, ones with a large symmetry group) one can obtain better approximability/computability results due to special features of the polynomials $p$ (for example, invariance with respect to the action of a large symmetry group). Indeed, the construction of the proof of Theorem 1.1 (see Section 2) preserves, for example, group invariance.

\section{Proof of Theorem 1.1}

Let $B$ be the unit ball of $\|\cdot\|$, so

$$
B=\{x \in V:\|x\| \leq 1\} .
$$

Hence $B$ is a centrally symmetric convex compact set containing the origin in its interior.

Let $V^{*}$ be the dual space of all linear functions $f: V \longrightarrow \mathbb{R}$ and let $C \subset V^{*}$ be the polar of $B$ :

$$
C=\left\{f \in V^{*}: f(x) \leq 1 \quad \text { for all } \quad x \in B\right\} .
$$

Hence $C$ is a centrally symmetric convex compact set containing the origin in its interior. Using the standard duality argument (see, for example, Section 1.6 of [3]), we can write

$$
\|x\|=\max _{f \in C} f(x) .
$$


Let

$$
W=V^{\otimes n}=\underbrace{V \otimes \ldots \otimes V}_{n \text { times }} \quad \text { and } \quad W^{*}=\left(V^{\otimes n}\right)^{*}=\underbrace{V^{*} \otimes \ldots \otimes V^{*}}_{n \text { times }}
$$

be the $n$-th tensor powers of $V$ and $V^{*}$ respectively.

For vectors $x \in V$ and $f \in V^{*}$ let

$$
x^{\otimes n}=\underbrace{x \otimes \ldots \otimes x}_{n \text { times }} \quad \text { and } \quad f^{\otimes n}=\underbrace{f \otimes \ldots \otimes f}_{n \text { times }}
$$

denote the $n$-th tensor power $x^{\otimes n} \in W$ and $f^{\otimes n} \in W^{*}$ respectively.

By (2.1), we can write

$$
\|x\|^{n}=\max _{f \in C}(f(x))^{n}=\max _{f \in C} f^{\otimes n}\left(x^{\otimes n}\right) .
$$

Let $D$ be the convex hull of $f^{\otimes n}$ for $f \in C$ :

$$
D=\operatorname{conv}\left\{f^{\otimes n}: f \in C\right\} .
$$

Then $D$ is a convex compact centrally symmetric (we use that $n$ is odd) subset of $W^{*}$ and from (2.2) we can write

$$
\|x\|^{n}=\max _{f \in C} f^{\otimes n}\left(x^{\otimes n}\right)=\max _{g \in D} g\left(x^{\otimes n}\right) .
$$

Let us estimate the dimension of $D$. There is a natural action of the symmetric group $S_{n}$ in $W^{*}$ which permutes the factors $V^{*}$, so that

$$
\sigma\left(f_{1} \otimes \ldots \otimes f_{n}\right)=f_{\sigma^{-1}(1)} \otimes \ldots \otimes f_{\sigma^{-1}(n)} .
$$

Let $\operatorname{Sym}\left(W^{*}\right) \subset W^{*}$ be the symmetric part of $W^{*}$, that is, the invariant subspace of that action. As is known, the dimension of $\operatorname{Sym}\left(W^{*}\right)$ is that of the space of homogeneous polynomials of degree $n$ in $d$ real variables (see, for example, Lecture 6 of [2]). Next, we observe that $f^{\otimes n} \in \operatorname{Sym}\left(W^{*}\right)$ for all $f \in V^{*}$ and, therefore,

$$
\operatorname{dim} D \leq \operatorname{dim} \operatorname{Sym}\left(W^{*}\right)=\left(\begin{array}{c}
n+d-1 \\
n
\end{array}\right)
$$

Let $E$ be the John's ellipsoid of $D$ in the affine hull of $D$, that is the (unique) ellipsoid of the maximum volume inscribed in $D$. As is known, (see, for example, Lecture 3 of [1])

$$
E \subset D \subset(\sqrt{\operatorname{dim} D}) E
$$

Combining this with (2.3), we write

$$
\max _{g \in E} g\left(x^{\otimes n}\right) \leq\|x\|^{n} \leq(\sqrt{\operatorname{dim} D}) \max _{g \in E} g\left(x^{\otimes n}\right)
$$


and, by (2.4),

$$
\max _{g \in E} g\left(x^{\otimes n}\right) \leq\|x\|^{n} \leq\left(\begin{array}{c}
n+d-1 \\
n
\end{array}\right) \max _{g \in E}^{\frac{1}{2}} g\left(x^{\otimes n}\right) .
$$

Let

$$
q(x)=\max _{g \in E} g\left(x^{\otimes n}\right) .
$$

We claim that $p(x)=q^{2}(x)$ is a polynomial in $x$ of degree $2 n$. Indeed, let us choose a basis $e_{1}, \ldots, e_{d}$ in $V$ and the dual basis $f_{1}, \ldots, f_{d}$ in $V^{*}$, so that $f_{i}\left(e_{j}\right)=\delta_{i j}$. Then $W$ acquires the basis

$$
e_{i_{1} \ldots i_{n}}=e_{i_{1}} \otimes \ldots \otimes e_{i_{n}} \quad \text { for } \quad 1 \leq i_{1}, \ldots, i_{n} \leq d
$$

and $W^{*}$ acquires the basis

$$
f_{i_{1} \ldots i_{n}}=f_{i_{1}} \otimes \ldots \otimes f_{i_{n}} \quad \text { for } \quad 1 \leq i_{1}, \ldots, i_{n} \leq d
$$

Geometrically, $V$ and $V^{*}$ are identified with $\mathbb{R}^{d}$ and $W$ and $W^{*}$ are identified with $\mathbb{R}^{d n}$. Let $K \subset W^{*}$ be the Euclidean unit ball defined by the inequality

$$
K=\left\{h \in W^{*}: \sum_{1 \leq i_{1}, \ldots, i_{n} \leq d} h_{i_{1} \ldots i_{n}}^{2} \leq 1\right\}
$$

where $h_{i_{1} \ldots i_{n}}$ is the corresponding coordinate of $h$ with respect to the basis $\left\{f_{i_{1} \ldots i_{n}}\right\}$. Since $E$ is an ellipsoid, there is a linear transformation $T: W^{*} \longrightarrow W^{*}$ such that $T(K)=E$. Let $T^{*}: W \longrightarrow W$ be the conjugate linear transformation and let $y=T^{*}\left(x^{\otimes n}\right)$. Hence the coordinates $y_{i_{1} \ldots i_{n}}$ of $y$ with respect to the basis $\left\{e_{i_{1} \ldots i_{n}}\right\}$ are polynomials in $x$ of degree $n$. Then

$$
\begin{aligned}
q(x) & =\max _{g \in E} g\left(x^{\otimes n}\right)=\max _{h \in K} T(h)\left(x^{\otimes n}\right)=\max _{h \in K} h\left(T^{*}\left(x^{\otimes n}\right)\right) \\
& =\max _{h \in K} h(y)=\sqrt{\sum_{1 \leq i_{1}, \ldots, i_{n} \leq d} y_{i_{1} \ldots i_{n}}^{2}} .
\end{aligned}
$$

Hence we conclude that $p(x)=q^{2}(x)$ is a homogeneous polynomial in $x$ of degree $2 n$, which is non-negative for all $x \in V$ (moreover, $p(x)$ is seen to be a sum of squares). From (2.5), we conclude that

$$
p^{\frac{1}{2 n}}(x) \leq\|x\| \leq\left(\begin{array}{c}
n+d-1 \\
n
\end{array}\right)^{\frac{1}{2 n}} p^{\frac{1}{2 n}}(x),
$$

as claimed. 


\section{An Extension to Minkowski Functionals}

There is a version of Theorem 1.1 for Minkowski functionals of not necessarily centrally symmetric convex bodies.

(3.1) Theorem. Let $V$ be a d-dimensional real vector space, let $B \subset V$ be a convex compact set containing the origin in its interior and let $\|x\|=\inf \{\lambda>0: x \in \lambda B\}$ be its Minkowski functional. For any odd integer $n>0$ there exist a homogeneous polynomial $p: V \longrightarrow \mathbb{R}$ of degree $2 n$ and a homogeneous polynomial $r: V \longrightarrow \mathbb{R}$ of degree $n$ such that $p(x) \geq 0$ and

$$
(r(x)+\sqrt{p(x)})^{\frac{1}{n}} \leq\|x\| \leq\left(r(x)+\left(\begin{array}{c}
n+d-1 \\
n
\end{array}\right) \sqrt{p(x)}\right)^{\frac{1}{n}}
$$

for all $x \in V$.

Proof. The proof follows the proof of Theorem 1.1 with some modifications. Up to (2.4) no essential changes are needed (note, however, that now we have to use that $n$ is odd in (2.2)). Then, since the set $D$ is not necessarily centrally symmetric, we can only find an ellipsoid $E$ (centered at the origin) of $W^{*}$ and a point $w \in D$, such that

$$
E \subset D-w \subset(\operatorname{dim} D) E
$$

see, for example, Lecture 3 of [1]. Then (2.5) transforms into

$$
\max _{g \in E} g\left(x^{\otimes n}\right) \leq\|x\|^{n}-w\left(x^{\otimes n}\right) \leq\left(\begin{array}{c}
n+d-1 \\
n
\end{array}\right) \max _{g \in E} g\left(x^{\otimes n}\right) .
$$

Denoting

$$
p(x)=\left(\max _{g \in E} g\left(x^{\otimes n}\right)\right)^{2} \quad \text { and } \quad r(x)=w\left(x^{\otimes n}\right)
$$

we proceed as in the proof of Theorem 1.1.

\section{REFERENCES}

1. K. Ball, An elementary introduction to modern convex geometry, in: Flavors of Geometry, 1-58, Math. Sci. Res. Inst. Publ., 31, Cambridge Univ. Press, Cambridge, 1997.

2. W. Fulton and J.Harris, Representation Theory. A First Course, Graduate Texts in Mathematics, 129, Readings in Mathematics, Springer-Verlag, New York, 1991.

3. R. Schneider, Convex Bodies: the Brunn-Minkowski Theory, Encyclopedia of Mathematics and its Applications, 44, Cambridge University Press, Cambridge, 1993.

Department of Mathematics, University of Michigan, Ann Arbor, Mi 48109-1109

E-mail address: barvinok@umich.edu 